\title{
Atuação dos Residentes Multiprofissionais em Saúde: percepções de trabalhadores dos serviços de saúde mental
}

\author{
Performance of Multiprofessional Residents in Health: perceptions of mental health service \\ workers
}

Desempeño de los residentes multiprofesionales en salud: percepciones de los trabajadores del servicio de salud mental

Janaina Lunardi Canabarro ${ }^{1 *}$, Marlene Gomes Terra ${ }^{1}$, Keity Laís Siepmann Soccol², Daiana Foggiato de Siqueira ${ }^{1}$.

\section{RESUMO}

Objetivo: Conhecer as percepções de trabalhadores dos serviços de atenção à saúde mental sobre a atuação dos residentes multiprofissionais em saúde. Métodos: Estudo qualitativo com 17 trabalhadores que atuam nos serviços de atenção à saúde mental. Utilizou-se a entrevista aberta para a coleta de dados, bem como a técnica de análise de conteúdo para organização e interpretação. Resultados: Os residentes multiprofissionais em saúde trazem melhorias ao cuidado em saúde, promovem a integração dos serviços e capacitam os trabalhadores nas suas práticas assistenciais. Ainda, os residentes fazem parte do processo de trabalho nos cenários onde estão inseridos. Conclusão: Evidenciou que os residentes multiprofissionais contribuem com o processo de trabalho no serviço de atuação pois trazem melhorias ao cuidado em saúde. Uma vez que eles realizam encaminhamento dos usuários a rede de atenção à saúde, promovem a integração dos serviços e capacitam os trabalhadores nas suas práticas assistenciais.

Palavras-chave: Prática profissional, Internato não Médico, Enfermagem.

\begin{abstract}
Objective: To know the perceptions of mental health care workers about the performance of multiprofessional residents in health. Methods: Qualitative study with 17 workers who work in mental health care services. The open interview was used for data collection, as well as the content analysis technique for organization and interpretation. Results: Multiprofessional health residents bring improvements in health care, promote the integration of services and train workers in their care practices. Also, residents are part of the work process in the scenarios where they are inserted. Conclusion: it evidenced that the multiprofessional residents contribute to the work process in the actuation service because they bring improvements to health care. Once they refer users to the health care network, promote the integration of services and train workers in their care practices.
\end{abstract}

Key words: Professional practice, Internship nonmedical, Nursing.

\section{RESUMEN}

Objetivo: Conocer las percepciones de los trabajadores de salud mental sobre el desempeño de los residentes multiprofesionales en salud. Métodos: Estudio cualitativo con 17 trabajadores que trabajan en servicios de salud mental. La entrevista abierta se utilizó para la recopilación de datos, así como la técnica de

1 Universidade Federal de Santa Maria (UFSM), Santa Maria-RS. *E-mail: jana.I.c2010@hotmail.com

2 Universidade Franciscana, Santa Maria-RS. 
análisis de contenido para la organización y la interpretación. Resultados: los residentes de salud multiprofesionales aportan mejoras en la atención médica, promueven la integración de servicios y capacitan a los trabajadores en sus prácticas de atención. Además, los residentes son parte del proceso de trabajo en los escenarios donde se insertan. Conclusión: se evidenció que los residentes multiprofesionales contribuyen al proceso de trabajo en el servicio de actuación porque aportan mejoras a la atención médica. Una vez que refieren a los usuarios a la red de atención médica, promueven la integración de servicios y capacitan a los trabajadores en sus prácticas de atención.

Palabras clave: Práctica profesional, Internado no médico, enfermeria.

\section{INTRODUÇÃO}

Em virtude das demandas de práticas assistenciais e a busca de atender a integralidade dos sujeitos envolvidos no processo saúde doença foram criados os Programas de Residência Multiprofissional em Saúde. Estes programas se mostram em expansão no país e estão se concretizando devido ao seu formato de aprendizagem, agregando ensino e serviço, formando profissionais qualificados com foco no trabalho multiprofissional frente a realidade social, política e cultural que está inseridos (MARTINS GM, et al., 2016).

Desta forma, os profissionais integrantes do Programa de Residência Multiprofissional em Saúde constituem as equipes de saúde, desenvolvendo ações de Educação Permanente em Saúde, incentivando a construção interdisciplinar e a reorientação das lógicas tecnoassistenciais (SILVA CTD, et al., 2014). Assim, faz-se necessário que as oportunidades de formação sejam ampliadas com vistas a atender às demandas sociais crescentes, fortalecendo a construção de um modelo assistencial pautado nos princípios que norteiam o Sistema Único de Saúde (SUS) (FORTE FDS, et al., 2016).

Os Residentes Multiprofissionais em Saúde estão inseridos em diversos cenários da saúde, dentre estes, os serviços de Saúde Mental. Nessa perspectiva, se faz importante refletir sobre as práticas em saúde mental com base na reformulação instituída pela Reforma Psiquiátrica. Sendo estes cenários dirigidos a atender a proposta de compreender o ser humano em seu processo existencial, abarcando o usuário em sua existênciasofrimento, nas suas diversas dimensões e não mais somente sob a espera de um diagnóstico. Mediante este pressuposto, a saúde mental, busca atender ao usuário prestando um cuidado humanizado, como capaz de colaborar no seu processo de reinserção social e consolidar cada vez mais o processo da Reforma Psiquiátrica (PESSOA JM, et al., 2016).

Diante da produção científica existente em torno da temática, foi realizada uma pesquisa nas bases de dados Literatura Latino-Americana e do Caribe em Ciências da Saúde (LILACS), Medical Literature Analysisand Retrieval System Online (MEDLINE) e Base de Dados da Enfermagem (BDENF), acerca da inserção de residentes multiprofissionais em serviços de saúde. Uma das lacunas evidenciadas foi à necessidade de escutar os trabalhadores de saúde dos serviços nos quais os Residentes Multiprofissionais em Saúde estão alocados, pois a formação deles ocorre por meio da sua inserção nesses locais.

Alicerçado nas reflexões envolvendo a temática, tem-se como questão de pesquisa: quais as percepções de trabalhadores dos serviços de atenção à saúde mental sobre a atuação dos residentes multiprofissionais em saúde? E, como objetivo: conhecer as percepções de trabalhadores dos serviços de atenção à saúde mental sobre a atuação dos residentes multiprofissionais em saúde.

\section{MÉTODOS}

Estudo qualitativo, desenvolvido nos serviços de atenção à saúde mental nos quais estão inseridos os profissionais residentes da Residência Multiprofissional em Saúde. Sendo assim, configuram-se cenários deste estudo: Unidade de Internação Psicossocial de um Hospital de Ensino e os Centros de Atenção Psicossocial (CAPS): CAPS II (transtornos mentais) e dois CAPS Álcool e Drogas. Estes serviços estão localizados em um município do estado do Rio Grande do Sul, Brasil. 
Esses serviços foram selecionados como cenário para o desenvolvimento do estudo por serem campos de atuação dos profissionais integrantes da Residência Multiprofissional em Saúde contemplado no Programa de Residência Multiprofissional em Saúde Mental no Sistema Público de Saúde. É importante informar que a ordem do serviço para iniciar a coleta de dados foi sorteada manualmente, de modo que a pesquisa contemplasse trabalhadores de todos os cenários citados anteriormente.

Foram convidados os trabalhadores das equipes de saúde mental dos CAPS e da Unidade de Internação Psicossocial. Os critérios de inclusão dos participantes deste estudo foram: trabalhadores da área da saúde de nível médio e superior, concursados e contratados pelo município, que estivessem atuando nos serviços de atenção à saúde mental durante o período da produção dos dados. Já os critérios de exclusão foram: trabalhadores afastados do serviço por motivo de licença a tratamento de saúde.

A definição dos participantes deu-se a partir de uma lista contendo os trabalhadores dos serviços de saúde, preenchida junto aos setores administrativos, levando em conta os critérios de inclusão e exclusão. De posse desta lista, realizou-se um sorteio manual, que foi realizado pela própria pesquisadora. Na sequência, os trabalhadores foram contatados pessoalmente ou via telefone, acerca da possibilidade de participarem da pesquisa.

A produção dos dados ocorreu de fevereiro a abril de 2016, por meio da entrevista que é uma ferramenta utilizada em pesquisas, uma vez que permite ao próprio participante refletir acerca de sua realidade, evidenciando ideias, crenças, opiniões, sentimentos e condutas, maneiras de pensar, de agir e de atuar. A entrevista é utilizada geralmente na descrição de casos individuais, na compreensão de especificidades culturais para determinados grupos (MINAYO MCS, 2014), que, no caso deste estudo são 17 trabalhadores de saúde dos serviços de saúde mental (dois assistentes sociais, dois auxiliares em assistência, quatro enfermeiros, dois fisioterapeutas, dois médicos, dois psicólogos, dois técnicos de enfermagem e um técnico em saúde mental), 12 são mulheres e cinco são homens, com idades entre 25 e 05 que trabalham cerca de um a cinco anos nesses serviços.

As entrevistas com os trabalhadores foram agendadas conforme as suas disponibilidades. Para tanto, foram realizadas nos referidos serviços, em uma sala reservada, de modo a manter um ambiente confortável, bem como preservando a identidade, a privacidade e a confidencialidade das informações fornecidas pelos participantes. O tempo de duração da entrevista não foi predeterminado, mas buscou respeitar a disponibilidade do trabalhador e duraram entre 20 à 60 minutos.

As entrevistas foram individuais, gravadas em áudio e transcritas na integra que se iniciou com a seguinte questão: como você percebe a inserção dos profissionais integrantes da Residência Multiprofissional nos cenários de saúde mental? Para a análise dos dados, foi utilizada a Análise Temática seguindo a Proposta Operativa de Minayo (MINAYO MCS, 2014), os dados obtidos foram relacionados e discutidos com diferentes autores que tratam da temática.

Da análise das entrevistas emergiram duas categorias, sendo elas: inserção dos residentes nos serviços de saúde mental e processo de trabalho dos residentes nos serviços de saúde mental. O estudo foi aprovado pelo comitê de ética em pesquisa da Universidade Federal de Santa Maria sob no 1.500.318. Ressalta-se que os trabalhadores concordaram com a participação na pesquisa assinando o Termo de Consentimento Livre e Esclarecido (TCLE), em duas vias, ficando uma com a pesquisadora responsável e outra com o participante. Os mesmos foram identificados pela letra "T", por ser a inicial da palavra 'trabalhadores', seguida de um número arábico (T1, T2, T3, e, assim sucessivamente).

\section{RESULTADOS}

\section{Inserção dos residentes nos serviços de saúde mental}

Os trabalhadores percebem que foi importante a inserção dos residentes na Residência Multiprofissional nos serviços de saúde mental para melhorar o cuidado prestado ao usuário e a integração dos serviços que compõem a Rede de Atenção Psicossocial. Acreditam que a residência capacita os residentes para serem bons profissionais em saúde mental e futuramente retornarem aos serviços como integrantes da equipe: 
"Fomos acolhendo a residência com braços abertos e pensando que deveríamos ajudar eles, e que teríamos várias possibilidades para a melhora do serviço, para melhorar a integração do serviço". (T8)

"Eu percebo como importante. Ela (Residência Multiprofissional em Saúde) dá aquele toque a mais, ela busca um profissional melhor que vai atender o paciente melhor. Sempre tem uma atividade a mais que as de rotina. Tem, também, toda essa questão social do paciente, que nos ajudou bastante e, tomara que eles nunca saiam daqui. Mudou bastante depois da inserção da residência". (T10)

A equipe de trabalhadores, por vezes, manifestou resistência quanto à inserção dos residentes nos serviços, devido ao aumento das responsabilidades dos trabalhadores com as atividades de ensino.

"Logo que a residência chegou havia uma resistência maior da equipe em relação à residência. Quando foi inserido (Residência Multiprofissional em Saúde) não participava de reuniões de equipe. Então, a equipe era mais fechada, distante da residência". (T1)

"No momento que eles (residentes) chegam aqui eu entendo que as funções são as mesmas e que tem muito preconceito ao estagiário, manda o residente ele é um "mandalete". Não! O residente ele é um profissional". (T7)

"Foi bem complicado quando a residência começou. Teve resistência da parte médica [..] não sei se foi por vaidade ou porque os dois queriam ter mais espaços ou muitas vezes não saber o que iriam fazer ali" (T10)

Assim, apesar de os residentes contribuírem e qualificarem a assistência prestada pelos serviços, existe certa resistência de alguns trabalhadores, dificultando a adesão dos residentes nos serviços, como se pode visualizar nas falas:

"Os serviços foram surpreendidos e esses outros campos também, talvez não soubéssemos muito como se dá essa questão teórica da inserção da residência, tanto que fecharam o campo logo. Eu acho que foi no primeiro semestre da residência que fecharam campo". (T9)

"Então, as pessoas não tinham uma boa recepção dela (Residência Multiprofissional em Saúde) e na verdade até hoje o trabalho dos residentes não tem uma boa adesão". (T11)

\section{Processos de trabalho dos residentes nos serviços de saúde mental}

Os trabalhadores perceberam que os residentes fazem parte dos processos de trabalho do cenário no qual estão inseridos.

Os residentes potencializam os trabalhadores a repensarem suas práticas, trazendo melhorias para o serviço e para o fortalecimento da equipe, bem como desacomodam os processos de trabalhos e os trabalhadores:

\footnotetext{
"A residência acabou de certa forma impulsionando e potencializando para que nós pensássemos sobre nossas práticas. Eles fazem parte dos processos de trabalho. Ela desacomoda os processos de trabalho e desacomoda os profissionais. Acaba de certa forma modificando esses processos de trabalho, fazendo com que os profissionais passem a refletir sobre algumas coisas". (T1)

"A residência complementa muito bem nesse sentido de ajudar repensar os processos trabalho a reorganizar dando um suporte, uma ajuda". (T3)

"Essa inserção de profissionais [...] é de suma importância para os processos. $A$ assistência de saúde é modificada e melhorada". (T6)
} 
Além disso, os participantes evidenciaram que os residentes proporcionam troca de experiências com a equipe, desenvolvem atividades em grupos com os usuários e conseguem implementar novos grupos devido ao olhar crítico e pela formação que recebem. Têm um amadurecimento, crescimento e uma formação crítica muito boa.

"Os residentes acabam tendo muito contato com os usuários. O que eu vejo da equipe é que conhece todo mundo e eles estão reconhecendo a participação dos residentes. Então, eles acabam se aproximando mais, fazendo mais grupo". (T3)

"Eles (residentes) trazem experiências epistemologicamente diferentes, corroborando em atividades de atendimento e acabam trazendo melhorias, pois demarcam várias coisas." (T5)

Os trabalhadores percebem que a equipe se fortaleceu, pois os residentes multiprofissionais gerenciam as suas ações de atendimento à demanda e dão continuidade nos processos de trabalho. Da mesma forma, eles dão conta de realizar o encaminhamento do usuário na rede, bem como de acompanhar situações após alta:

"Estão sempre bem envolvidos com esses grupos de dança, em grupos de políticas públicas e da horta [...] nós aprendemos com eles e eles aprendem com nós. [...] Eu vejo eles como uma complementação da nossa força de trabalho, porque, às vezes, faltam profissionais e nós sabemos que podemos contar com eles". (T4)

"Então, estamos tentando esse movimento. Tentando fazer com que as coisas tomem um novo rumo. [...] Para que possamos ter uma melhor condição de trabalho e dar continuidade a esses processos de trabalho". (T8)

"Eles acompanham inclusive o encaminhamento para rede. E, após a alta também para saber o estado de saúde desse paciente. [...] Eles sempre se comunicam sobre o que vai ser realizado, o que está acontecendo com o paciente [...]. Eles sempre nos dão retorno dos pacientes, até para poder passar essa confiança". (T12)

\section{DISCUSSÃO}

Convém destacar que o desenvolvimento desta pesquisa apresenta algumas limitações, como o tempo em que foi desenvolvida e a dificuldade de as pessoas estarem implicadas na continuidade da formação profissional. Por isso, não se almeja generalizar os resultados, contudo, sua contribuição está no aprofundamento da temática abordada e na compreensão da inserção da Residência Multiprofissional em Saúde nos serviços de Saúde Mental.

Considera-se que este estudo possui relevância aos trabalhadores da área da saúde, pois poderá proporcionar a reflexão sobre suas práticas de cuidado e a sua compreensão acerca do papel e ações desenvolvidas pelos residentes. Ainda, possibilitará a valorização potencial dos residentes diante de sua atuação e contribuição para a formação de profissionais que construam práticas comprometidas com a concepção de saúde recomendada pelo SUS. Dessa forma, os trabalhadores poderão compreender a relevância da atuação da Residência Multiprofissional em Saúde e do trabalho em equipe nos serviços de saúde mental, minimizando o desafio enfrentado na inserção dos residentes.

A inserção da Residência Multiprofissional em Saúde nos serviços permite a vivência da formação e a articulação de campo e núcleo de saber. O profissional em formação se prepara para atuar em equipe e compartilha essa responsabilidade de atuar conforme os princípios e diretrizes do SUS, trazendo melhoras para o cuidado em saúde. Sendo importante que ele conheça a pluralidade das linhas de intervenções e o território no seu contexto de atuação, onde se valoriza o vivido e as relações que ali acontecem (CEZAR PK, et al., 2015).

Ainda, a inserção dos residentes permite a integração ensino-serviço, a qual se faz necessária a participação ativa e o acolhimento da equipe. A integração ensino-serviço é um dos instrumentos que tem por 
finalidade a reorientação da formação dos profissionais, privilegiando aprendizagens por meio de troca entre o profissional e o residente, a qual proporciona a concretização das novas formas de fazer saúde (COSTA AC e AZEVEDO CC, 2016). Estes compartilham do planejamento e da estruturação dos serviços e, assim, gerenciam o que estão fazendo. Desta forma, constroem o conhecimento de forma integrada e coletiva diante de um contexto prático, exercendo a análise crítica reflexiva.

Nesse sentido, a formação da residência passa por um processo de imersão no campo, possibilitando a articulação com a teoria. Para que se alcance êxito nos resultados, faz-se necessário a integração ensinoserviço, bem como a atuação multiprofissional. Esta é a proposta dos Programas de Residência Multiprofissional em Saúde, pois busca articular a prática e a formação como uma estratégia de mudança, que inspira ações as quais têm impactado no processo de trabalho das equipes e nos cursos de formação. Além disso, recomenda unir temáticas de pesquisa que emergiram nas experiências (CEZAR PK, et al., 2015).

A Residência Multiprofissional em Saúde caracteriza-se por ser modalidade de pós-graduação lato sensu onde o treinamento em serviço se estabelece como uma maneira efetiva de aprendizado e especialização na área da saúde, além de permitir a integração entre ensino, serviço e comunidade (MIRANDA NETO MV, ET AL., 2015). Assim, o programa possibilita a relação de troca de conhecimentos entre as distintas especialidades e entre o saber científico e o saber popular, pois o profissional está imerso nos serviços, articulando teoria e prática e contribuindo para a atenção à saúde e para os serviços de saúde (MELLO AL, et al., 2018).

A atuação multiprofissional necessita estar estabelecida entre todos os integrantes para que seja alcançado o objetivo proposto das ações e intensifique o cuidado prestado ao usuário. Para isto, a equipe multiprofissional realiza acompanhamento e identifica ganhos nas condições de saúde dos usuários (MAIA DB, et al., 2013). Neste sentido, a Residência Multiprofissional em Saúde estimula e desencadeia os processos de aprendizado, pois oferece suporte aos trabalhadores, por meio da capacitação e atualização da equipe.

Essas ações de compartilhamento de saberes e troca de experiências servem como um meio de transformação das práticas a partir de seu cotidiano de trabalho. A formação profissional nos cenários de saúde diante da consolidação do SUS é desafiante tanto para as instituições formadoras, que disponibilizam programas de graduação e pós-graduação, quanto às instituições prestadoras de serviços de saúde em suas ideações de significar os espaços do trabalho como espaços de aquisição de conhecimento (ENGSTROM EM e MOTTA JIV, 2016).

O fato de haver residentes para atuar nas práticas de saúde mental foi fundamental para as ações desenvolvidas pela equipe. Em contrapartida, alguns profissionais, são resistentes e não observaram mudanças ou contribuições da inserção dos residentes nas práticas assistenciais. Isto se justifica pela falta de clareza quanto ao objetivo da residência, dificultando a avaliação sobre suas ações (GARCIA SD, et al., 2014).

Tal resistência por parte de alguns trabalhadores dificulta a adesão dos residentes nos serviços. Dessa maneira, a integração com a equipe constitui uma das metas a serem alcançadas pela coordenação e residentes do Programa. Todavia é importante sinalizar que avanços existem. A integração das equipes na atenção aos usuários dos serviços impulsiona novas conquistas (CHEADE MFM, et al.,2013).

Em relação à inserção dos residentes multiprofissionais, percebeu-se a possibilidade de melhoria no serviço, de modo que a Residência Multiprofissional em Saúde seja bem acolhida pelos trabalhadores dos serviços de saúde mental. Nesse sentido, é importante salientar que a compreensão do papel dos residentes nos serviços de saúde mental, bem como as suas participações no processo de inclusão, diminui a resistência por parte dos trabalhadores. Os residentes fazem parte dos processos de trabalho onde potencializam os trabalhadores a repensarem suas práticas, trazendo melhorias para o serviço e para o fortalecimento da equipe. Eles são instigados a participarem, junto aos trabalhadores de campo, de atividades educativas que envolvam toda a equipe de saúde, bem como, auxiliar no aprimoramento da equipe em suas atividades diárias (GARCIA SD, et al., 2014). 
A Residência Multiprofissional em Saúde é fundamental, pois permite que os trabalhadores dos serviços de saúde descubram novos horizontes e preencham as fragilidades das ações de cuidado em saúde (CHEADE MFM, et al.,2013). Os residentes modificam os processos de trabalho no serviço permitindo aos trabalhadores repensarem suas ações e refletirem sobre elas. Além disso, possibilitam a troca de experiências entre trabalhadores dos serviços e permitem que eles apreendam novos métodos de trabalho.

A troca de experiências entre profissionais dos serviços e os residentes permite a ambos apreenderem novos métodos de trabalho. A possibilidade da troca de saberes acontece por meio de atividades executadas e planejadas em equipe, promovendo a interdisciplinaridade, a integralidade e humanização nas ações em saúde (CEZAR PK, et al., 2015).

$O$ reconhecimento de que todos ensinam e aprendem é fundamental para que se atinja significado e modificações no âmbito de trabalho (GARCIA SD, et al., 2014). Ao adentrar em um espaço, aprende-se acerca das rotinas, experiências e vivenciais de trabalhadores que estão inseridos ali. É a partir deste aprendizado que são aplicados os conhecimentos científicos e empíricos aprendidos em um determinado.

Assim sendo, trabalhar em equipe na residência é uma excelente oportunidade de aprendizado, de aproximação com a equipe, além de favorecer a troca de conhecimento e experiências com profissionais das demais áreas. Conforme já referido, a Residência Multiprofissional em Saúde pode ser considerada um dispositivo que proporciona melhorias na formação dos residentes, diminuindo os desafios encontrados na formação acadêmica, pois após sua inserção no Programa de Residência Multiprofissional em Saúde ampliase a oportunidade de trabalhar engajados em um conceito de saúde ampliado (SILVA CT, et al., 2016). Dessa forma, os Programas de Residência Multiprofissional em Saúde permite a formação de um novo profissional, com visão ampliada de saúde, atentando para uma prática assistencial mais crítica, tendo um olhar atento e crítico às implicâncias e às especialidades de cada pessoa, e, sobretudo, entendendo o princípio de resolutividade do SUS (MAIA DB, et al., 2013).

A Residência Multiprofissional em Saúde desencadeia reflexões frente a prática assistencial nos serviços de saúde. Nesse sentido, utiliza métodos inovadores quanto ao modo de conduzir os processos educativos e a promoção da saúde em grupo. Para que a população a ser atingida se torne componente ativo no processo de aprendizado de fazer saúde, faz-se necessário estimular e propor uma relação prazerosa de interação que implica benefícios à saúde física, mental e social (SERBIM AK, et al., 2013).

Nessa acepção, a Residência Multiprofissional em Saúde atua em seus espaços de trabalho como instrumento de atualização para a equipe de saúde, que estão em constante processo de formação, devido à articulação teoria/prática (GARCIA SD, et al., 2014). Desenvolvem atividades que proporcionam os espaços educativos. Nesta perspectiva de formação, a residência modifica os processos de trabalho, desacomodando a equipe, uma vez que permeia a troca de saberes e construção coletiva de conhecimento e desacomoda a equipe.

Conforme apontado pelos participantes, os residentes assumem, em alguns momentos, a demanda das atividades existentes no serviço, como se fossem trabalhadores da equipe. Isso vai ao encontro de um estudo desenvolvido que teve como objeto os residentes, os quais afirmaram que são produtores de trabalho e, muitas vezes, não conseguem participar de espaços para questionamentos de suas indagações com a equipe de saúde (FERNANDES MNS, et al., 2015).

Ainda, no processo de trabalho, os residentes realizam encaminhamentos dos usuários dentro dos serviços de saúde. Esses são de acordo com a necessidade do cuidado e da competência inerente a sua formação (SILVA JC, et al., 2015). Sabe-se que o encaminhamento estimula a integração de núcleos profissionais que oportuna à excelência na execução das práticas assistenciais e gera ganho ao paciente, uma vez que ele pode ser atendido de acordo com seus valores culturais, intelectuais e sociais.

Os residentes fazem parte dos processos de trabalho nos serviços saúde mental, onde realizam atividades que promovem a integração com os trabalhadores, permite a troca de experiência e a apreensão de conhecimento devido ao seu olhar crítico inerente a formação que recebe. Além disso, desacomoda os 
servidores em relação aos seus processos de trabalho, visto que são instigados a pensarem e a refletirem sobre as suas atuações como profissionais. E, também atendem demandas do serviço bem como, realizam encaminhamentos dos usuários para a rede de saúde, trazendo melhorias para assistência. Entretanto, dependem da compreensão de cada trabalhador e isso é importante para que o cuidado em saúde seja aprimorado.

Os residentes constituem os processos de trabalho dos serviços de saúde mental, onde fomentam a reflexão das práticas do cuidado, atendem demanda, propiciam melhorias para o serviço e o fortalecimento da equipe. Além disso, eles potencializam as trocas de experiências com a equipe e implementam novos grupos no serviços devido ao olhar crítico e pela boa formação que recebem. Ainda, eles desenvolvem atividades multiprofissionais e desacomodam a equipe por meio análise crítica reflexiva.

\section{CONCLUSÃO}

Evidenciou-se que os residentes multiprofissionais contribuem de modo significativo no processo de trabalho e para a capacitação da equipe de enfermagem. Os residentes nos serviços trazem melhorias ao cuidado em saúde, uma vez que realiza encaminhamento dos usuários a rede de atenção à saúde, promove a integração dos serviços e capacita os trabalhadores nas suas práticas assistenciais. Porém, alguns trabalhadores consideram que essa inserção ocassiona impactos desfavoráveis nos serviços, pois desconhecem a proposta da Residência Multiprofissional em Saúde o que dificultava o entrosamento e a integração entre eles.

\section{REFERÊNCIAS}

1. CEZAR PK, RODRIGUES PM, ARPINI DM. A Psicologia na Estratégia de Saúde da Família: Vivências da Residência Multiprofissional. Psicol Cienc Prof, 2015; 35(1):211-24.

2. CHEADE MFM, FROTA OP, LOUREIRO MDR et al. Residência multiprofissional em saúde: a busca pela integralidade. Cogitare Enferm, 2013; 18(3):734-40.

3. COSTA AC, AZEVEDO CC. The Teaching-Service Integration and the Multiprofessional Residency in Health: an experience report at a Basic Health. Tempus, actas de saúde colet, 2016; 10(4):265-282.

4. ENGSTROM EM, MOTTA JIV. Training of professionals in post-graduation courses in public health and primary healthcare in the municipality of Rio de Janeiro, Brazil. Ciênc Saúde Coletiva, 2016; 21(5):1461-70.

5. FERNANDES, MNS, BECK CLC, WEILLER THVV et al. Suffering and pleasure in the process of forming multidisciplinary health. Rev Gaúcha Enferm, 2015; 36(4):90-7.

6. FORTE FDS, MORAIS HG, RODRIGUES SAG et al. Educação interprofissional e o programa de educação pelo trabalho para a saúde/Rede Cegonha: potencializando mudanças na formação acadêmica. Interface (Botucatu), 2016; 20(58):787-96.

7. GARCIA SD, IGNOTTI BS, CICILIATO CZ et al. The meaning of nursing internship to interns at a public university. Rev Enferm UERJ, 2014; 22(2):212-18.

8. MAIA DB, SOUZA ETG, GAMA RM et al. Atuação interdisciplinar na atenção básica de saúde: a inserção da residência multiprofissional. Sau Transf Soc, 2013; 4(1):103-10.

9. MARTINS GM, CAREGNATO RCA, BARROSO VLM et al. Implementation of multiprofessional healthcare residency at a federal university: historical trajectory. Rev Gaúcha Enferm, 2016; 37(3): e57046.

10. MELLO AL, TERRA MG, NIETSCHE EA et al. Formação de Residentes Multiprofissionais em Saúde: Limites e Contribuições para a Integração Ensino-Serviço, 2018; 8:e2567.

11. MINAYO MCS. O desafio do Conhecimento. Pesquisa qualitativa em saúde. 14.ed. São Paulo: Hucitec; 2014.

12. MIRANDA NETO MV, LEONELLO VM, OLIVEIRA MAC. Multiprofessional residency in health: a document analysis of political pedagogical projects Residencias multidisciplinarios en salud: análisis documental de los proyectos político-pedagógicos. Rev Bras Enferm, 2015; 68(4):586-93.

13. PESSOA JM, SANTOS RCA, CLEMENTINO FS, OLIVEIRA KKD, MIRANDA FAN. Mental health policy in the context of psychiatric hospitals: Challenges and perspectives. Esc Anna Nery, 2016; 20(1):83-89.

14. SERBIM AK, GERLACK LF, MARCHI DSM. Oficinas Multiprofissionais: Educação em Saúde para Idosos de uma Comunidade. Rev Eletr Gestão Saúde, 2013; 4(11):1500-10.

15. SILVA CT, TERRA MG, KRUSE MHL et al. Multi-professional residency as an intercessor for continuing education in health. Texto Contexto Enferm, 2016; 25(1): e2760014.

16. SILVA CTD, TERRA MG, CAMPONOGARA $S$ et al. Permanent health education based on research with professionals of a multidisciplinary residency program: case study. Rev Gaúcha Enferm, 2014; 35(3):49-54.

17. SILVA JC, CONTIM D, OHL RI et al. Perception of the residents about their performance in the multidisciplinary residency program. Acta Paul Enferm, 2015; 28(2):132-8. 